\title{
PET/MR attenuation correction: where have we come from and where are we going?
}

\author{
Dimitris Visvikis • Florian Monnier • Julien Bert • \\ Mathieu Hatt • Hadi Fayad
}

Published online: 15 March 2014

(C) Springer-Verlag Berlin Heidelberg 2014

The advent of clinical multimodality imaging with the development of PET/CT scanners [1] has presented us with ample opportunities to harvest the benefits of combining functional and anatomical imaging. These benefits concern improved PET quantitative accuracy and overall patient management (improved diagnostic accuracy and therapy response assessment), but also increased patient throughput. It is indeed the last of these points that has substantially contributed to the rapid acceptance of PET/CT imaging in clinical practice eclipsing PET-only systems. Indeed, one of the major reasons behind the improved patient throughput achieved with PET/ $\mathrm{CT}$ has been the use of $\mathrm{CT}$ images for attenuation correction (AC) of the acquired PET emission datasets. In this context, $\mathrm{CT}$ images possess two desirable properties. Firstly, CT acquisitions are very fast, removing the need for long radionuclide-based transmission imaging that was traditionally used in PET (about $50 \%$ of the overall acquisition times). Secondly, CT intensity values represent the attenuation properties of the tissues in the imaging field of view, albeit at X-ray photon energies. The necessary transformation of CT images into attenuation maps at $511 \mathrm{keV}$ can be achieved by bilinear transformation [2]. Although such a transformation represents a certain approximation, CT-based AC of PET datasets using such attenuation maps has been shown to lead to the same level of quantitative accuracy and superior contrast in the

D. Visvikis $\cdot$ F. Monnier $\cdot$ J. Bert $\cdot$ M. Hatt $\cdot$ H. Fayad INSERM, UMR1101 LaTIM, CHRU Brest, Brest, France

F. Monnier $\cdot$ H. Fayad

Universite de Bretagne Occidentale, Brest, France

D. Visvikis $(\square)$

LaTIM, INSERM UMR1101, CHRU MORVAN,

5 avenue Foch, 29609 Brest, France

e-mail: dimitris@univ-brest.fr reconstructed PET images compared to radionuclide-based transmission scanning [3].

In the last couple of years clinical PET/MR devices have become a reality and the first results concerning the potential of this modality in terms of patient management are beginning to emerge. However, different issues persist with respect to the quantitative accuracy of this new modality, concerning in particular the question of PET AC based on the use of MRderived attenuation maps. A recent study published in this journal clearly reinforces these issues for neurological imaging [4]. In contrast to CT imaging, MRI does not provide direct information concerning tissue attenuation properties, and there is therefore no direct way to obtain the required information for PET AC purposes. Most of the approaches currently proposed in clinical PET/MR systems for PET AC are based on the combination of specific MR sequences and subsequent image segmentation.

Amongst these, the approach implemented in the first generation of clinical systems is based on the two-point Dixon gradient echo sequence [5]. This sequence, which involves a few seconds of acquisition time, allows the separation of water and fat tissue by using the chemical shift of fat relative to that of water. This information facilitates in turn the segmentation of MR images into four to five different classes (lung, fat tissue, nonfat tissue, mixture of fat/nonfat tissue, air) [6]. It is important to highlight that once segmented, fixed $511 \mathrm{keV}$ linear attenuation coefficients (LACs) are assigned to each of the considered tissue types, largely ignoring tissue heterogeneities. However, the biggest drawback of this approach is the lack for consideration of bone structures which are considered as soft tissue for the purpose of reconstructing PET AC maps. The use of this approach may therefore introduce severe quantitative errors depending clearly on the location of the region of interest. In terms of quantitative accuracy, it is generally accepted that the inclusion of bone in the AC of brain PET images is essential. On the other hand, the exclusion 
of bone structures in whole-body imaging introduces quantitative errors mostly in the case of osseous lesions. Compared to CT-based AC in whole-body PET/MR imaging, standardized uptake value underestimation may vary from a few percent up to $30 \%$ depending not only on the lesion location but also on the composition of bone lesions [7]. This result further highlights the need to use a continuous scale in the LACs rather than a fixed value assigned to segmented tissue regions. In the case of brain imaging, a recent study published in EJNMMI [4] has also shown variable mean regional activity concentration underestimation of $10-22 \%$ compared to CT-based AC, with the smaller differences measured in structures such as the striatum, thalamus and hippocampus.

In order to account for and improve the overall accuracy of segmentation based AC, more recent implementations of PET $\mathrm{AC}$ in clinical PET/MR consider the use of an ultrashort echo time (UTE) sequence [8]. UTE sequences have been proposed in MRI for the visualization of bone which has a very short spin-spin relaxation time T2. UTE-based AC involves acquisitions at two echo times, one visualizing bone, while the signal for other tissue types is the same in both images. Different methodologies have been subsequently proposed in order to provide a three-tissue class (air, soft tissue, bone) segmentation approach for PET AC [9] or alternatively to use a triple-echo sequence combining UTE and Dixon to distinguish four tissue classes (air, soft tissue, bone, fat tissue) [10]. These approaches have been almost exclusively evaluated in brain imaging showing mean activity concentration differences in the entire brain of about $5 \%$ relative to CT-based $\mathrm{AC}$, although maximum differences could be up to $20-40 \%$ [8-10]. Similarly, a more detailed study on a region by region basis, considering 25 patients undergoing FDG PET of the brain, has shown that despite a decrease in the measured mean activity underestimation resulting from the use of a threetissue class UTE-based approach compared to Dixon-based AC, substantial underestimations compared to CT-based AC still occur. These differences (up to an average of $20 \%$ ) are also region-dependent, with the worst results at the level of the cerebellum which is located at the level of the sinuses, where the mixture of air, soft tissue and bone structures represents a substantial challenge for all approaches, including UTE based. In general terms average percentage differences of $10-15 \%$ relative to $\mathrm{CT}$-based $\mathrm{AC}$ are measured in the frontal, temporal and parietal lobes. This regional variability in the measured differences throughout the brain is a clear issue for neurological applications. Furthermore, there seems to be a lack of standardization with respect to the UTE protocols for AC currently in use both in terms of overall acquisition times and selection of individual parameters. On the other hand, there are only a few reports on the use of UTE sequences in whole-body MR imaging, since their application is hampered by long acquisition times and field inhomogeneities associated with an extended field of view. Therefore, the extension of this approach to whole-body imaging represents a real challenge.

There is therefore a clear need for improved AC in PET/ MR not only for neurological but also whole-body imaging applications. This improvement should be both in terms of the accuracy in determining the spatial extent of the structures of interest and in the use of attenuation maps with continuous LACs. There are different approaches based on the use of the atlas combined with machine learning techniques that have been proposed in order to improve both of these aspects. The basic idea behind these approaches is to explore a database of paired CT and MR patient images. These images in combination with the acquired MR datasets for a given patient are subsequently used to derive a patient-specific pseudo-CT map. Another advantage of these approaches is that in principle they can provide attenuation maps with continuous LACs, eliminating issues associated with the use of single tissue values that do not account for tissue heterogeneities. Different MR sequences can be considered in the MR-CT paired datasets used in the atlas in order to improve the overall accuracy of the identified structures of interest. One of these approaches uses a combination of atlas-derived information and pattern recognition to obtain patient-specific pseudo-CT maps [11]. Evaluation of this approach in brain images showed average activity concentration differences of $<4 \%$ relative to $\mathrm{CT}$-based $\mathrm{AC}$ in different brain areas, but interregional differences were not reported.

A clear issue with any atlas-based approach for brain and whole-body applications is the accurate handling of pathology, interpatient lung density variations, and the presence of metallic implants. In an attempt to improve overall robustness, a modified version of this same approach including atlasbased artefact detection has recently been applied to wholebody imaging and has led to mean activity underestimations of $<6 \%$ [12]. Variants of this approach consider the use of multiple MR sequences to improve the identification of different tissues classes and hence to improve the overall atlas registration process and the subsequent pseudo-CT prediction model [13, 14]. Although such alternatives have only been tested in brain imaging, since they mostly use UTE sequences, they are clearly associated with longer MR acquisition times which may compromise their clinical utility in PET/MR. What is currently missing is large-scale clinical evaluation studies of these atlas and machine-learning approaches that would clearly demonstrate their robustness with respect to the presence of anatomical abnormalities which are largely patient-specific and as such hard to account for in any atlas-based approach.

One has finally to consider the truncation issues associated with MR-based AC maps which can be important in wholebody imaging given that the patients are scanned with arms down because of multiple practical issues. Different solutions have already been implemented in current generation PET/ MR devices based on either information from uncorrected 
PET images [15] or on the use of a modified iterative maximum likelihood reconstruction of attenuation and activity for estimating the missing part of the attenuation map from the PET emission data [16]. Despite the lack of resolution in bone structures in the arms, both approaches have been reported to reduce errors to $<5 \%$, but clearly larger scale clinical studies are necessary to demonstrate their performance and associated robustness in clinical practice. On the other hand, both approaches will clearly benefit from time of flight $(\mathrm{ToF})$ information to further improve their accuracy.

An alternative that has more recently emerged is the use of non-MR information for PET AC in PET/MR. The first such approach is based on the use of transmission scanning within the PET/MR device [17], using radionuclide sources and the acquisition of both emission and transmission datasets during the PET acquisition. In order to be able to carry out such simultaneous acquisitions, the PET device requires ToF capability. Although theoretically feasible, the question of limited space available with combined PET/MR devices poses certain associated engineering challenges for the clinical implementation of such an approach, which may therefore be more appropriate for sequential PET/MR systems only. The second option is based on purely exploring information inherent in the acquired PET emission datasets relating to tissue attenuation without the need for any explicit transmission data acquisition. If one assumes that the true emission data distribution is known there will be only a single attenuation map that can be consistent with that emission distribution and can be therefore estimated. However, the problem is poorly determined and previous attempts have led to poor results with emission data structures contained in the estimated AC maps. More recently Defrise et al. [18] have demonstrated that the spatial constraints provided by the ToF information in the emission datasets may allow more robust exploitation of the consistency conditions to determine the attenuation images from the acquired emission datasets. Despite the fact that current devices are limited in terms of ToF resolution to $400-500 \mathrm{ps}$, this study also showed that this ToF resolution is sufficient to obtain good results for clinical applications. Although this initial proof of principle work was performed in 2-D using an analytical algorithm, current studies in this active field are concentrating on an extension to 3-D and the use of iterative algorithms that allow better noise modelling [19].

In conclusion, the development of combined PET/MR devices has brought to the forefront of scientific interest the issue of PET AC, after CT largely contributed to its solution in multimodality PET/CT imaging. Current clinical AC implementations are moving towards the inclusion of bone structures which are clearly essential in quantitative neurological PET imaging, in principle one of the flagship applications of PET/MR. However, substantial quantitative differences compared to CT-based AC persist, which appear to be also region-dependent with the largest differences in areas in the vicinity of dense bone and/or a substantial mixture of bone, air and soft tissue. There is now clearly a need for studies in larger patient populations with protocol standardization in the MR sequence parameters used in order to further evaluate these latest developments for quantitative brain imaging in clinical practice. Atlas and machine-learning approaches offer the possibility to correct for attenuation including bone structures both in brain and whole-body PET/MR applications. However, these approaches need to be further assessed in clinical practice in order to demonstrate their robustness in the imaging of different patient-specific pathology types. Finally, the calculation of attenuation maps directly from emission datasets is the most promising solution for future ToF-based PET/MR devices.

\section{References}

1. Beyer T, Townsend DW. Putting 'clear' into nuclear medicine: a decade of PET/CT development. Eur J Nucl Med Mol Imaging. 2006;33(8):857-61.

2. Burger C, Goerres G, Schoenes S, Buck A, Lonn AH, Von Schulthess GK. PET attenuation coefficients from CT images: experimental evaluation of the transformation of CT into PET $511-\mathrm{keV}$ attenuation coefficients. Eur J Nucl Med Mol Imaging. 2002;29(7):922-7.

3. Visvikis D, Costa DC, Croasdale I, Lonn AHR, Bomanji J, Gacinovic S, et al. CT based attenuation correction in the calculation of semi-quantitative indices of $18 \mathrm{FDG}$ uptake in PET. Eur J Nucl Med Mol Imaging. 2003;30(3):344-53.

4. Dickson JC, O'Meara C, Barnes A. A comparison of CT- and MRbased attenuation correction in neurological PET. Eur J Nucl Med Mol Imaging. 2014. doi:10.1007/s00259-013-2652-z.

5. Coombs BD, Szumowski J, Coshow W. Two-point Dixon technique for water-fat signal decomposition with B0 inhomogeneity correction. Magn Reson Med. 1997;38:884-9.

6. Martinez-Moller A, Souvatzoglou M, Delso G, Bundschuh RA, Chefd'hotel C, Ziegler S, et al. Tissue classification as a potential approach for attenuation correction in whole body PET/MRI: evaluation with PET/CT data. J Nucl Med. 2009;50(4):520-6.

7. Samarin A, Burger C, Wollenweber SD, Crook DW, Burger IA, Schmid DT, et al. PET/MR imaging of bone lesions - implications for PET quantification from imperfect attenuation correction. Eur J Nucl Med Mol Imaging. 2012;39:1154-60.

8. Keereman V, Fierens Y, Broux T, De Deene Y, Lonneux M, Vandenberghe S. MRI based attenuation correction for PET/MRI using ultrashort echo time (UTE) sequence. J Nucl Med. 2010;51: $812-8$.

9. Catana C, van der Kouwe A, Benner T, Michel CJ, Hamm M, Fenchel M, et al. Toward implementing an MRI-based PET attenuation-correction method for neurologic studies on the MRPET brain prototype. J Nucl Med. 2010;51(9):1431-8.

10. Berker Y, Franke J, Salomon A, Palmowski M, Donker HCW, Temur $\mathrm{Y}$, et al. MRI-based attenuation correction for hybrid PET/MRI systems: a 4-class tissue segmentation technique using a combined ultrashort-echo-time/Dixon MRI sequence. J Nucl Med. 2012;53: 796-804.

11. Hofmann M, Steinke F, Scheel V, Charpiat G, Farquhar J, Aschoff P, et al. MRI-based attenuation correction for PET/MRI: a novel approach combining pattern recognition and atlas registration. $\mathrm{J}$ Nucl Med. 2008;49(11):1875-83. 
12. Bezrukov I, Schmidt H, Mantlik F, Schwenzer N, Brendle C, Schölkopf B, et al. MR-based attenuation correction methods for improved PET quantification in lesions within bone and susceptibility artifact regions. J Nucl Med. 2013;54(10):1768-74.

13. Johansson A, Karlsson M, Nyholm T. CT substitute derived from MRI sequences with ultrashort echo time. Med Phys. 2011;38(5): 2708-14.

14. Navalpakkam BK, Braun H, Kuwert T, Quick HH. Magnetic resonance-based attenuation correction for PET/MR hybrid imaging using continuous valued attenuation maps. Invest Radiol. 2013;48: 323-32.

15. Delso G, Martinez-Möller A, Bundschuh RA, Nekolla SG, Ziegler SI. The effect of limited MR field of view in MR/PET attenuation correction. Med Phys. 2010;37(6):2804-12.
16. Nuyts J, Bal G, Kehren F, Fenchel M, Michel C, Watson C. Completion of a truncated attenuation image from the attenuated PET emission data. IEEE Trans Med Imaging. 2013;32(2): 237-46.

17. Mollet P, Keereman V, Clementel E, Vandenberghe S. Simultaneous MR-compatible emission and transmission imaging for PET using time-of-flight information. IEEE Trans Med Imaging. 2012;31(9): 1734-42.

18. Defrise M, Rezaei A, Nuyts J. Transmission-less attenuation correction in time-of-flight PET: analysis of a discrete iterative algorithm. Phys Med Biol. 2014;59(4):1073-95.

19. Defrise M, Rezaei A, Nuyts J. Time-of-flight PET data determine the attenuation sinogram up to a constant. Phys Med Biol. 2012;57(4): 885-99. 